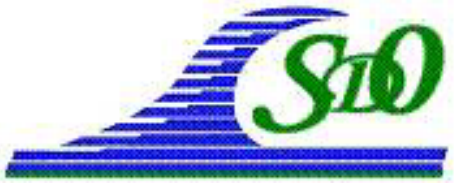

Xìmes Journées Nationales Génie Côtier - Génie Civil

Les Sables d'Olonne, 22-25 juin 2010

DOI:10.5150/jngcgc.2010.035-G C Editions Paralia CFL

disponible en ligne - http://www.paralia.fr - available online

\title{
Morphogenèse des bancs jouxtant les caps sous l'influence combinée de la marée et de la houle
}

\author{
Nicolas GUILLOU ${ }^{1}$, Georges CHAPALAIN ${ }^{1}$
}

1. Laboratoire de Génie Côtier et Environnement,

Centre d'Etudes Techniques Maritimes Et Fluviales, 155 rue Pierre Bouguer,

Technopôle Brest-Iroise, BP 5, 29280 Plouzané, France.

nicolas.guillou@developpement-durable.gouv.fr

georges.chapalain@developpement-durable.gouv.fr

\section{Résumé :}

De grands bancs longs de 5 à $30 \mathrm{~km}$ et larges de 1 à $2 \mathrm{~km}$ tendent à se former au voisinage des caps dans des environnements tidaux sableux. Ces structures sédimentaires sont qualifiées de bancs en drapeau ("banner banks"). Leur morphologie est variée, depuis des bancs uniques jusqu'à des bancs doubles plus ou moins symétriques. La présente étude s'attache à examiner les effets de la houle dans la genèse des bancs en drapeau en appliquant la chaîne de modélisation numérique MISTRAL couplant des modules hydrodynamiques de circulation tridimensionnelle de marée et de génération, propagation et dissipation de houle et des modules de transport sédimentaire et d'évolution morphologique. Elle concerne le cas schématique d'un cap de forme gaussienne dont les dimensions correspondent à celles du cap de Gay Head (Massachussets). Les résultats des simulations montrent une influence de la houle sur la morphogenèse des bancs en (i) asymétrisant leur développement de part et d'autre de la pointe du cap et (ii) réorientant l'axe des bancs le long du flanc du cap.

\section{Mots-clés :}

Bancs en drapeau - Interaction houle-courant - Transport sédimentaire - Charriage Suspension - Evolution morphologique

\section{Introduction}

Les bancs en drapeau ("banner banks") se forment au voisinage des caps dans des environnements tidaux sableux. Leur longueur varie de 5 à $30 \mathrm{~km}$ pour une largeur comprise entre 1 et $2 \mathrm{~km}$. Leur morphologie est variée, depuis des bancs uniques jusqu'à des bancs doubles plus ou moins symétriques.

Cette asymétrie a fait l'objet de plusieurs études. PINGREE (1978) l'impute à l'action de la force de Coriolis. Selon sa théorie, la résultante du gradient de pression est plus importante dans le cas des tourbillons cycloniques et favorise l'accumulation des sédiments en leurs centres. SIGNELL \& HARRIS (2000) réfutent cette hypothèse sur la base d'une modélisation numérique aboutissant au développement de bancs 
symétriques. BERTHOT \& PATTIARATCHI (2006) ont récemment avancé la nécessité d'inclure les effets de la houle dans la genèse des bancs en drapeau.

La présente étude s'attache à examiner cette dernière hypothèse via l'application de la chaîne de Modélisation Intégrée pour la Simulation des TRAnsports Littoraux MISTRAL couplant des modules hydrodynamiques de circulation tridimensionnelle (3D) de marée et de génération, propagation et dissipation de houle et des modules de transport sédimentaire en charriage et en suspension et d'évolution morphologique. Elle concerne le cas schématique d'un cap de forme gaussienne initialement proposé par SIGNELL \& GEYER (1991).

L'article s'organise selon les étapes suivantes. Après une description succincte des codes numériques et des conditions de simulation (section 2), les résultats des évolutions morphologiques sont présentés pour une situation de courant de marée seul et différentes conditions de houle variant en amplitudes et directions (section 3). Enfin, la section 4 synthétise ces résultats.

\section{Modèles numériques et conditions de simulation}

\subsection{Modèles numériques}

La modélisation de l'hydrodynamique repose sur le couplage des codes de circulation 3D COHERENS ("COupled Hydrodynamical-Ecological model for RegioNal and Shelf seas", LUYTEN et al., 1999) et de propagation spectrale de houle SWAN ("Simulating WAves Nearshore", BOOIJ et al., 1999). Le couplage est effectué via un module dédié au calcul des interactions entre les couches limites de houle et de courant près du fond. Basé sur la théorie de GRANT \& MADSEN (1979), ce module intègre les modifications induites sur le profil vertical de viscosité turbulente près du fond, la contrainte de cisaillement totale et le frottement apparent ressenti par le courant à proximité du fond. Les courants de dérive associés à la houle ne sont pas pris en compte dans l'étude.

Les taux de transport en charriage sont déterminés de manière analytique à partir des formules de VAN RIJN (1984) en situation de courant de marée seul conformément aux travaux de SIGNELL \& HARRIS (2000) et de Soulsby-Van Rijn (SOULSBY, 1997) dans le cas d'une houle superposée à un courant de marée. Les concentrations de sédiment en suspension sont calculées via le module de transport scalaire de COHERENS adapté à la résolution de l'advection et de la dispersion 3D de particules sédimentaires dans la colonne d'eau. Les évolutions des fonds sont le résultat d'une équation de continuité intégrant la divergence des taux de transport en charriage et le bilan des flux de suspension de dépôt et d'érosion à l'interface eau-sédiment.

Des détails supplémentaires sur les développements numériques entrepris sont disponibles dans GUILLOU (2007). 


\subsection{Conditions de simulation}

Les conditions de simulation reprennent la configuration proposée par SIGNELL \& GEYER (1991) et inspirée du cas de Gay Head (Massachussetts) d'un cap de forme gaussienne de $8 \mathrm{~km}$ de long et $2 \mathrm{~km}$ de large (figure 1). Le cap est positionné au milieu de la frontière sud, considérée solide, d'un domaine rectangulaire de $50 \mathrm{~km}$ de long et $20 \mathrm{~km}$ de large ouvert à ses frontières ouest, nord et est. La profondeur varie linéairement vers le nord selon une pente de $0,67 \%$ pour atteindre $20 \mathrm{~m}$ à $3 \mathrm{~km}$ de la côte.

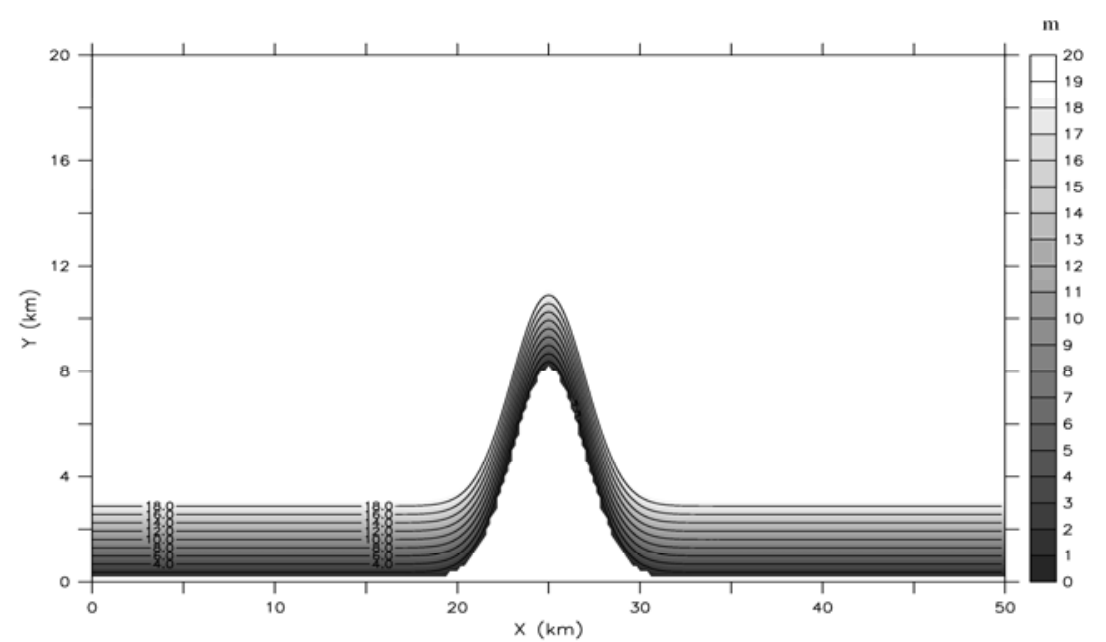

Figure 1. Bathymétrie du cas schématique d'un cap de forme gaussienne.

Le modèle hydrosédimentaire est mis en place sur ce domaine de calcul selon un maillage horizontal correspondant à une grille régulière de $200 \mathrm{~m}$ de pas d'espace. Le maillage vertical est fixé à 10 niveaux $\sigma$. Il est complété par un sous-maillage comportant 20 niveaux $\sigma$ de la demi-cellule verticale de fond pour un calcul optimisé du flux de dépôt des particules sédimentaires à l'interface eau-sédiment (GUILLOU, 2007). Les pas de temps des modes barotrope et barocline sont respectivement fixés à 5 et $60 \mathrm{~s}$. Des détails sur la paramétrisation des coefficients de diffusion et de viscosité turbulentes sont disponibles dans GUILLOU (2007). Les paramètres annexes du modèle reprennent les valeurs adoptées par SIGNELL \& HARRIS (2000) : un coefficient de Coriolis fixé à $\mathrm{f}=1,0 \times 10^{-4}$ et un paramètre de rugosité $\mathrm{z}_{0}=0,003 \mathrm{~m}$. Un courant alternatif sinusoïdal semi-diurne de $0,5 \mathrm{~m} \mathrm{~s}^{-1}$ d'amplitude est imposé le long de la frontière ouest. Une condition de radiation est appliquée le long de la frontière est. Une condition de glissement est utilisée à la frontière nord. La frontière sud est considérée comme un mur imperméable avec une condition de glissement sur les courants.

Le modèle de propagation de houle est appliqué à des emprises étendues centrées sur le domaine de calcul afin de s'astreindre de l'influence des frontières latérales. La résolution spatiale horizontale reste fixée à $200 \mathrm{~m}$. Les simulations sont effectuées en mode stationnaire avec une discrétisation de la densité de variance des vagues en (i) 60 
directions selon une résolution de $6^{\circ}$ et (ii) 30 fréquences réparties de 0,0566 à $1 \mathrm{~Hz}$ selon une progression géométrique. Le terme de dissipation par frottement sur le fond est paramétré selon MADSEN et al. (1988) avec un coefficient de rugosité $\mathrm{z}_{0}=0,003 \mathrm{~m}$. Enfin, quatre conditions limites de houle variant en amplitude et en direction sont testées (tableau 1). La période de pic est fixée à $10 \mathrm{~s}$ et l'étalement directionnel à $20^{\circ}$.

La formation des bancs par charriage est étudiée à partir d'une couverture sédimentaire uniforme de sables moyens de $250 \mu \mathrm{m}$ de diamètre, la formation des bancs par suspension avec une couverture de silts de $30 \mu \mathrm{m}$ de diamètre (SIGNELL \& HARRIS, 2000). Les évolutions morphodynamiques sont simulées pendant deux cycles de marée. Les simulations à plus long terme nécessitent d'intégrer les effets des évolutions bathymétriques dans les calculs hydrodynamiques.

Tableau 1. Conditions limites des houles incidentes.

\begin{tabular}{lllll}
\hline & Condition 1 & Condition 2 & Condition 3 & Condition 4 \\
\hline Hauteur $\boldsymbol{h}_{\boldsymbol{m} 0}$ & $0,3 \mathrm{~m}$ & $0,5 \mathrm{~m}$ & $1,0 \mathrm{~m}$ & $1,0 \mathrm{~m}$ \\
Direction & nord & nord & nord & nord-ouest \\
\hline
\end{tabular}

\section{Résultats numériques}

\subsection{Conditions de marée seule}

Les évolutions morphodynamiques obtenues au bout de deux cycles de marée (figure 2) s'accordent avec les résultats numériques de SIGNELL \& HARRIS (2000) avec :

- dans le cas du charriage (figure 2a), la progression d'une zone de dépôt dans le prolongement de la tête du cap et l'apparition de part et d'autre de l'axe central de deux zones érosives avec au large la présence des bancs,

- et dans le cas de la suspension (figure 2b), la mise en place d'une zone érosive au nord de la tête du cap et le développement de part et d'autre de cet axe de deux bancs symétriques.

Les évolutions symétriques des bancs confirment l'influence négligeable de la force de Coriolis dans la formation de ces structures réfutant la théorie proposée par PINGREE (1978). Dans le cas de la suspension, le développement des bancs est sensiblement plus important à l'est. SIGNELL \& HARRIS (2000) montrent que ce développement est lié à une faible atténuation de l'onde de marée au cours de sa propagation d'ouest en est.

Les évolutions morphodynamiques associées au charriage (figure 2a) sont localisées à proximité de la tête du cap. En condition de flot (considéré dirigé d'ouest en est dans la présente étude), l'accélération des courants le long du flanc amont conduit à une érosion locale et à l'apparition d'une zone de dépôt face à la tête du cap (figure 3a). Le développement d'une recirculation en aval lors de l'apparition du jusant scinde la zone de dépôt initiale en deux régions (figure 3-b). La première est située dans le 
prolongement du cap. La deuxième apparaît au large de la zone zone d'érosion. Un développement symétrique au cours du cycle de marée suivant accroît ces évolutions.
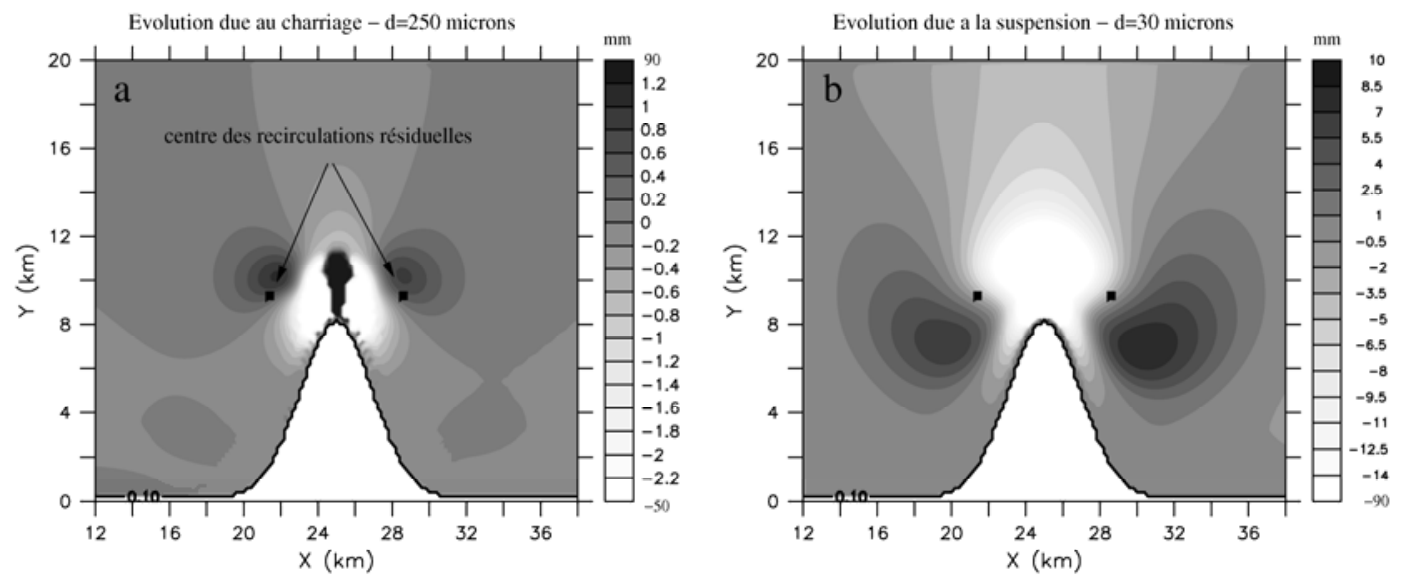

Figure 2. Evolutions morphodynamiques liées aux transports (a) par charriage et (b) par suspension au bout de deux cycles de marée dans le cas d'un courant de marée seul.
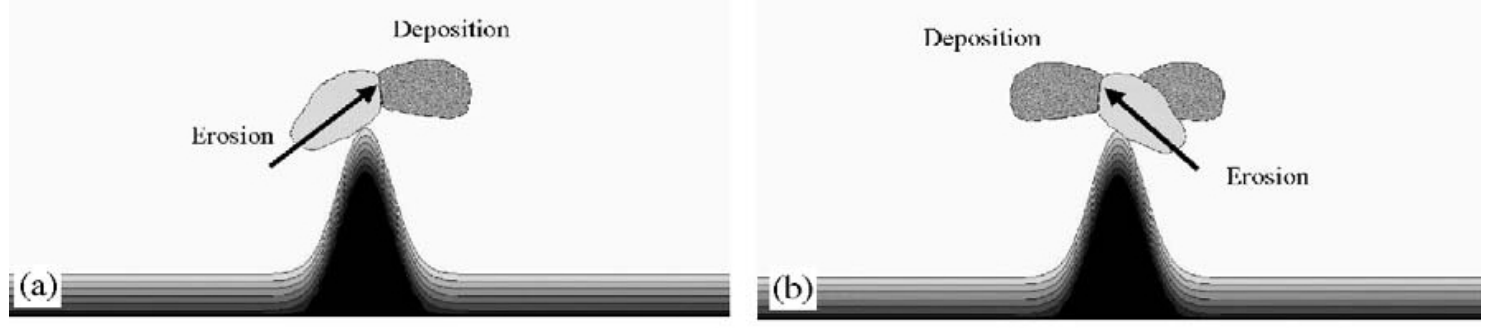

Figure 3. Schéma des processus conduisant à la formation des bancs par charriage au cours (a) du flot et (b) du jusant (d'après BERTHOT \& PATTIARATCHI, 2006).

Les évolutions morphodynamiques liées à la suspension (figure 2b) dépendent principalement de la contrainte de cisaillement locale. Les mises en suspension sont ainsi initialisées le long du flanc du cap. Les maxima sont atteints à la tête du cap conduisant à une érosion massive du lit sédimentaire dans cette région. Les zones de dépôt apparaissent au niveau des aires de décélération des courants situées de part et d'autre de l'axe central du cap et décalé de $3 \mathrm{~km}$ au sud du centre des recirculations résiduelles (figure $2 b$ ).

\subsection{Conditions de houle superposée à la marée}

La figure $4 a$ présente les évolutions morphodynamiques par charriage au bout de deux cycles de marée pour une houle de nord de $0,3 \mathrm{~m}$. Les différences d'évolution par rapport à cette simulation sont représentées sur les figures $4 \mathrm{~b}$ à $\mathrm{d}$ pour les trois conditions de houle restantes. L'amplitude de la houle incidente module l'évolution des bancs par charriage (figures $4 \mathrm{a}-\mathrm{c}$ ). Une houle de nord de 1,0 $\mathrm{m}$ induit ainsi une 
migration des bancs vers la côte méridionale parallèlement aux flancs du cap (figure 4c). Une houle de nord-ouest de 1,0 m (figure 4d) conduit à une évolution asymétrique avec :

- un développement du banc exposé à la houle 8 fois plus important que pour une houle de nord de même amplitude,

- une réduction de la hauteur du banc situé du côté protégé des houles pondérée d'une sensible augmentation au large de la zone de dépôt centrale.

En effet, l'érosion observée le long des flancs du cap conditionne le dépôt au niveau des bancs situés dans leur prolongement (figure 3). Dans le cas d'une houle de nord-ouest, une situation proche de la colinéarité entre la houle et les courants apparaît le long du flanc situé à l'est. La contrainte totale et les taux d'érosion résultants sont exacerbés, ce qui conduit à une augmentation des quantités de sédiment déposées dans le prolongement du flanc. La différence est accentuée avec le flanc ouest caractérisé par un cas de quadrature entre la houle et les courants.

Les évolutions morphodynamiques par suspension sont présentées à la figure 5 au bout de deux cycles de marée pour les quatre conditions de houle testées. Les houles de nord de 0,3 et $0,5 \mathrm{~m}$ (figures $5 \mathrm{a}$ et $\mathrm{b}$ ) ne modifient que très sensiblement la morphologie des bancs par rapport à une situation de courant de marée seul. Les modifications sont plus importantes pour une hauteur de 1,0 m (figure $5 \mathrm{c}$ ). Le banc se forme alors au large de la zone littorale selon un axe parallèle au flanc du cap. En effet, l'augmentation des contraintes de cisaillement au large favorisent, à proximité de la côte méridionale, le dépôt des sédiments érodés à la tête du cap et transportés par la recirculation créée en aval. Une condition de houle de nord-ouest induit un développement asymétrique du banc dans la zone protégée de la houle incidente (figure 5d).

\section{Conclusions}

Les simulations montrent l'impact significatif des houles de tempête sur le développement des bancs. Les houles induisent (i) une migration des bancs développés par charriage de part et d'autre de la tête du cap vers la côte méridionale et (ii) une formation des bancs par suspension au large de la zone littorale selon un axe parallèle au flanc du cap. Les houles d'incidence oblique favorisent le développement d'un seul banc du côté exposé dans le cas du charriage et le long du flanc protégé dans le cas de la suspension. Ces travaux se sont focalisés sur les effets du phénomène d'interaction houle-courant en couche limite de fond. Les perspectives de ce travail consisteront à étudier les impacts de l'effet Doppler des courants sur la houle et de la génération des courants de houle en faibles profondeurs. 

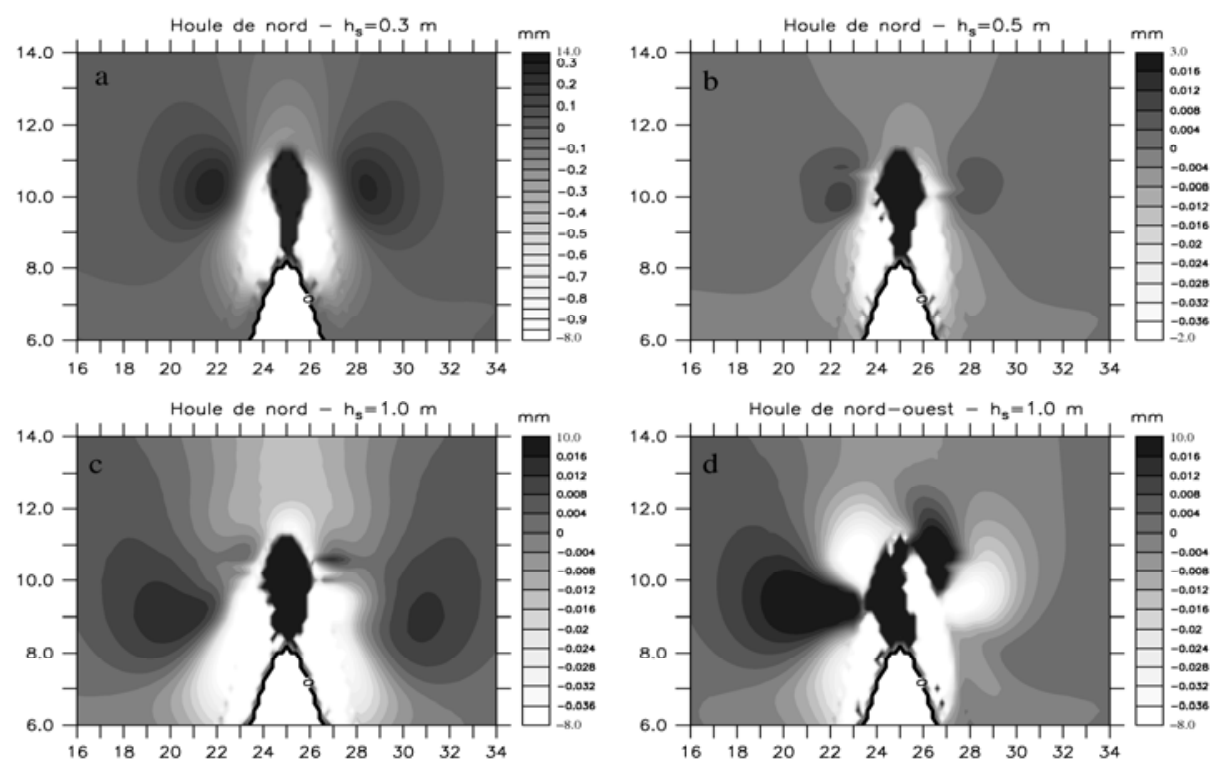

Figure 4. Evolutions morphodynamiques par charriage à proximité de la tête du cap au bout de deux cycles de marée pour une houle de nord de 0,3 m (a) et différentiels par rapport à ce cas pour une houle de nord de $0,5 \mathrm{~m}$ (b), de $1,0 \mathrm{~m}$ (c) et une houle de nord-ouest de $1,0 \mathrm{~m}(\mathrm{~d})$.
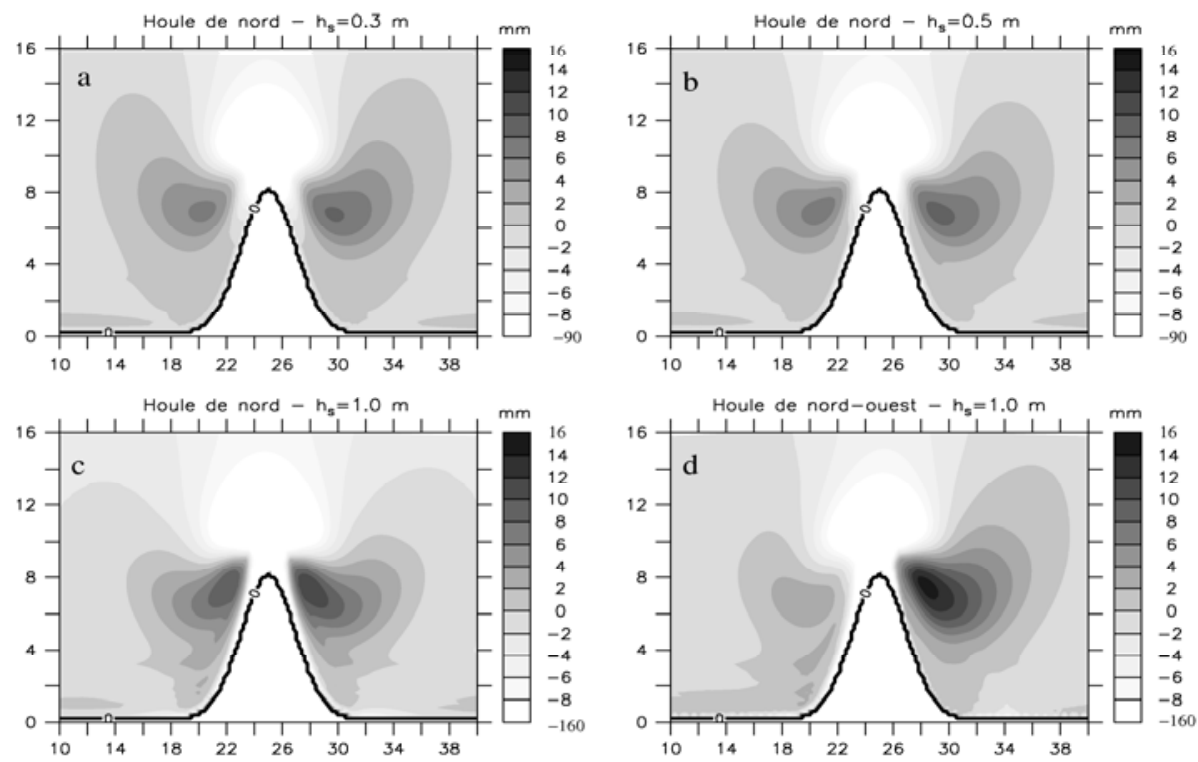

Figure 5. Evolutions morphodynamiques par suspension au bout de deux cycles de marée pour une houle de nord de 0,3 m (a), de 0,5 m (b) et 1,0 m (c) et une houle de nord-ouest de 1,0 $\mathrm{m}(\mathrm{d})$. 
Thème 2 -Dynamique sédimentaire et transports des particules

\section{Références bibliographiques}

BERTHOT A., PATTIARATCHI C.B. (2006). Mechanisms for the formation of headland-associated linear sandbanks. Continental Shelf Research, 26, pp 987-1004. doi:10.1016/j.csr.2006.03.004

BOOIJ N.R.C., RIS R.C., HOLTHUIJSEN L.H. (1999). A third generation wave model for coastal regions, part $i$, model description and validation. Journal of Geophysical Research, 104, pp 7649-7666. doi:10.1029/98JC02622

GRANT W.D., MADSEN O.S. (1979). Combined wave and current interaction with a rough bottom. Journal of Geophysical Research, C4, pp 1797-1808. doi:10.1029/JC084iC04p01797

GUILLOU N. (2007). Rôles de l'hétérogénéité des sédiments de fond et des interactions houle-courant sur l'hydrodynamique et la dynamique sédimentaire en zone subtidaleapplications en Manche orientale et à la pointe de la Bretagne. Thèse de doctorat de l'Université de Bretagne Occidentale, $469 \mathrm{p}$.

LUYTEN P.J., JONES J.E., PROCTOR R., TABOR A., TETT P., WILD-ADEN K. (1999). COHERENS: A Coupled Hydrodynamics-Ecological model for RegioNals and Shelf seas. Management Unit of the Mathematical Models of the North Sea, Belgium. MADSEN O.S., POON Y.K., GRABER H.C. (1988). Spectral wave attenuation by bottom friction. Theory. Proc. 21th Int. Conf. Coastal Engineering, ASCE, pp 492-504. PINGREE R.D. (1978). The formation of the Shambles and other banks by tidal stirring of the seas. Journal of the marine biological association, 58, pp 211-226.

SIGNELL R.P., GEYER C.K. (1991). Transient eddy formation around headlands. Journal of Geophysical Research, vol. 96, $\mathrm{n}^{\circ} \mathrm{C} 2$, pp 2561-2575. doi:10.1029/90JC02029

SIGNELL R.P., HARRIS C.K. (2000). Modeling sand bank formation around tidal headlands. In: Malcolm, S. (Ed.). Proceedings of the 6th Estuarine and Coastal Modeling Conference, ASCE Press, New York.

SOULSBY R.L. (1997). Dynamics of marine sand. Thomas Telford. H.R. Wallingford, $249 \mathrm{p}$.

VAN RIJN L.C. (1984). Sediment transport, part I: Bedload Transport. Journal of Hydraulic Division, ASCE, 10, 110, pp 1431-1456. doi:10.1061/(ASCE)07339429(1984)110:10(1431) 